\title{
Effects of antipsychotics on bone mineral density and prolactin levels in patients with schizophrenia: a 12-month prospective study
}

\author{
Mengxin Wang ${ }^{1}$, Ruihua $\mathrm{Hou}^{2}$, Jia Jian ${ }^{3}$, Guolin $\mathrm{Mi}^{3}$, Huimin $\mathrm{Qiu}^{3}$, Bingyu Cao ${ }^{3}$ and Maoqin Tang ${ }^{3} *$ \\ ${ }^{1}$ Shandong University Qilu Hospital, Jinan, Shandong, China \\ ${ }^{2}$ Department of Psychiatry, Clinical and Experimental Sciences, Faculty of Medicine, University of Southampton, Southampton, \\ United Kingdom \\ ${ }^{3}$ Shandong Mental Health Center, Jinan, Shandong, China
}

\begin{abstract}
Objective Effects of conventional and atypical antipsychotics on bone mineral density (BMD) and serum prolactin levels (PRL) were examined in patients with schizophrenia.

Methods One hundred and sixty-three first-episode inpatients with schizophrenia were recruited, to whom one of three conventional antipsychotics (perphenazine, sulpiride, and chlorpromazine) or one of three atypical antipsychotics (clozapine, quetiapine, and aripiprazole) was prescribed for 12 months as appropriate. BMD and PRL were tested before and after treatment. Same measures were conducted in 90 matched healthy controls.

Results Baseline BMD of postero-anterior L1-L4 range from 1.04 \pm 0.17 to $1.42 \pm 1.23$, and there was no significant difference between the patients group and healthy control group. However, post-treatment BMD values in patients (ranging from 1.02 \pm 0.15 to $1.23 \pm 0.10$ ) were significantly lower than that in healthy controls (ranging from $1.15 \pm 0.12$ to $1.42 \pm 1.36$ ). The BMD values after conventional antipsychotics were significantly lower than that after atypical antipsychotics. The PRL level after conventional antipsychotics $(53.05 \pm 30.25 \mathrm{ng} / \mathrm{ml}) \mathrm{was}$ significantly higher than that after atypical antipsychotics $(32.81 \pm 17.42 \mathrm{ng} / \mathrm{ml})$. Conditioned relevance analysis revealed significant negative correlations between the PRL level and the BMD values after conventional antipsychotics.

Conclusion The increase of PRL might be an important risk factor leading to a high prevalence of osteoporosis in patients with schizophrenia on long-term conventional antipsychotic medication. Copyright (c) 2014 John Wiley \& Sons, Ltd.
\end{abstract}

KEY WORDS - conventional/atypical antipsychotics; bone mineral density; prolactin; schizophrenia

\section{INTRODUCTION}

Schizophrenia is a chronic, severe, and disabling mental disorder. The prevalence of schizophrenia approaches $1 \%$, and the incidence is about 1.5 per 10,000 people (McGrath et al., 2008; Van Os and Kapur, 2009). Although the incidence is low, the prevalence is high due to chronicity. It currently affects 24 million people (World Health Organization, 2011) and is considered to be one of the top ten causes of long-term disability worldwide. In most cases, schizophrenia starts between the ages of 15 and 35 years, and approximately $75 \%$ of patients have ongoing disability with relapses; therefore, long-term use of antipsychotics is needed to achieve stable condition and reduce recurrence.

Osteoporosis is a systemic skeletal metabolic disorder characterized by a reduction in bone mineral

*Correspondence to: M. Tang, Department of Psychiatry, Shandong Mental Health Center, 49 Wenhua East Road, Jinan City, Shandong Province, China 250014. Tel: 0086-18764145579; Fax: 0086-531-88932855 E-mail: tangmaoqin2008@126.com density (BMD), which leads to an increased risk of fracture (World Health Organization, 1994; Alldredge et al., 2009). Research has shown that patients with schizophrenia have a higher incidence rate of developing osteoporosis; the mechanism underlying this is multifactorial and includes general as well as disease-specific factors, such as antipsychotic medication and hyperprolactinemia (Javaid and Holt, 2008). Therefore, the increasing awareness of the effect of antipsychotics on bone metabolism may prompt clinicians to screen patients at high risk of antipsychotic-induced osteoporosis and reduce the incidence of potentially avoidable fractures (Howard et al., 2007; Renn et al., 2009; Partti et al., 2010; Graham et al., 2011). Research has also indicated that some risk factors may contribute to the development of osteoporosis in patients with schizophrenia, such as inadequate intake of nutrition, excessive thirst, low activity, excessive smoking, and alcohol abuse. In addition, the occurrence of osteoporosis has been linked to the metabolism of antipsychotics. Such drugs usually need to be metabolized in the liver and kidney, and long-term usage can damage liver 
function, leading to a synthetic reduction of vitamin D, which promotes calcium absorption by the gastrointestinal system. In addition, the sedative effects of some antipsychotics reduce patients' outdoor activity, which further limits calcium absorption.

Antipsychotics are the most common cause of pharmacologic hyperprolactinemia (Molitch, 2005). Some conventional antipsychotics have been found to regulate prolactin secretion, whereas most atypical antipsychotics do not (Kaneda et al., 2004; Montejo, 2008). A study of 422 psychotic patients showed that antipsychotic therapy is strongly associated with hyperprolactinemia; in particular, a significantly higher prevalence of hyperprolactinemia was found in the conventional antipsychotics-treated patients compared with the atypical antipsychotics-treated patients group (Montgomery et al., 2004). Some recent research suggests that antipsychotic-related hyperprolactinemia may lead to osteoporosis and fragility fractures (Rey-Sánchez et al., 2009; Roke et al., 2009), whereas other studies reported contradictory findings (Lee et al., 2010; Renn et al., 2010; Sugawara et al., 2011). Therefore, the relationship between hyperprolactinemia and osteoporosis remains controversial, and further research is warranted.

This study aimed to investigate how long-term use of conventional and atypical antipsychotics affects spine BMD cross postero-anterior (PA) L1-L4 and how it is associated with PRL in patients with schizophrenia. This is the first study to compare the effects of two generations of antipsychotics on both BMD and PRL in a 12-month follow-up and provides important evidence for intervention strategies for osteoporosis in schizophrenia.

\section{METHODS}

\section{Participants}

All participants (aged between 25 and 45 years) who met the diagnostic criteria for schizophrenia based on the International Classification of Diseases 10th revision were recruited from Shandong Mental Health Center, China. They were all inpatients with first-episode schizophrenia during the period of recruitment (from May 2010 to April 2012), with a total score of $\geq 60$ on the Positive and Negative Symptoms Scale (PANSS). All patients had an educational level of primary school or above, which enabled them to understand study information and give informed consent. Individuals with serious neurological and physical diseases that may cause osteoporosis or bone metabolism and those with a history of alcohol or drug abuse were excluded.
Two hundred and forty eligible patients completed a questionnaire booklet regarding their sociodemographic status and clinical features. BMD measure of PA L1-L4, serum prolactin level (PRL), estrogen (E2), bone alkaline phosphatase (BALP), and crosslaps (CTX) were examined on the day of admission and again 12 months after antipsychotic treatment. All patients were randomly allocated to single dosage of the conventional antipsychotics group $(n=120)$ with 40 cases in each of the chlorpromazine, perphenazine, and sulpiride subgroups, and atypical antipsychotics group $(n=120)$ with 40 cases in each of the clozapine, quetiapine, and aripiprazole subgroups.

Ninety healthy controls were also recruited during the same period from the community in Jinan City, Shandong Province. Their age, gender, body mass index (BMI), marital status, and years of education matched those of the patients group.

The study protocol was approved by the Research Ethics Committee of Shandong University, and prior written informed consent was obtained from all participants.

\section{Medications-antipsychotics options}

Conventional antipsychotics: chlorpromazine $25 \mathrm{mg}$ per tablet manufactured by Beijing Yi Min Pharmaceuticals, perphenazine $2 \mathrm{mg}$ per tablet manufactured by Tianjin Lisheng Company, and sulpiride $100 \mathrm{mg}$ per tablet manufactured by Jiangsu Xuzhou Pharmaceuticals.

Atypical antipsychotic: clozapine $25 \mathrm{mg}$ per tablet manufactured by Jiangsu Yunyang Pharmaceuticals, quetiapine $200 \mathrm{mg}$ per tablet manufactured by Astrazeneca, and aripiprazole $5 \mathrm{mg}$ per tablet manufactured by Zhejiang Otsuka pharmaceuticals.

The use and dosage of antipsychotics were prescribed by consultant psychiatrists according to patients' conditions. The range and average dosage prescribed were chlorpromazine $300 \sim 600 \mathrm{mg} /$ day $(325.0 \pm 47.63 \mathrm{mg} /$ day $)$, perphenazine $16 \sim 48 \mathrm{mg} /$ day $(30.0 \pm 7.82 \mathrm{mg} /$ day), sulpiride $600 \sim 1200 \mathrm{mg} /$ day $(800.0 \pm 144.01 \mathrm{mg} /$ day $) ;$ clozapine $200 \sim 400 \mathrm{mg} /$ day $(318.75 \pm 21.10 \mathrm{mg} /$ day), quetiapine $400 \sim 750 \mathrm{mg} /$ day $(650.0 \pm 62.36 \mathrm{mg} / \mathrm{day})$, and aripiprazole $10 \sim 30 \mathrm{mg} /$ day $(18.25 \pm 7.86 \mathrm{mg} /$ day $)$. No other medications were prescribed except for a small dosage of benzodiazepine drugs ( $2 \sim 4 \mathrm{mg} / \mathrm{night})$ and benzhexol ( $2 \sim 8 \mathrm{mg} /$ day $)$.

\section{Measures}

Questionnaires. Psychotic symptoms were assessed by PANSS (Kay et al., 1988), which is a medical scale used for measuring symptom severity of patients with schizophrenia. It is widely used in the study of antipsychotic therapy. A general questionnaire was designed 
and used to collect demographic information such as age, gender, weight, educational level, economic situation, and marital status; and clinical data such as medical history, duration of disease, diagnostic classification, and current medications, which were obtained from medical records.

Measure of bone mineral density. The most widely validated technique, the dual-energy X-ray absorptiometry (by American General Medical Equipment Co.), was used to measure BMD. The BMD measure of PA $\mathrm{L} 1-\mathrm{L} 4\left(\mathrm{~g} / \mathrm{cm}^{2}\right)$ was detected by the application of dual-energy two-photon reuptake X-ray measurement, and the scanned image was based on the surface bone density of $1.001 \mathrm{~g} / \mathrm{cm}^{2}$. Quality assurance was carried out before assessment with a deviation of $0.46 \%$ and coefficients of variation of $0.08 \%$. According to the World Health Organization (WHO) criteria, osteoporosis is defined as a BMD that lies 2.5 SD or more below the average value for young healthy women (a t-score of $<-2.5 \mathrm{SD}$ ) (World Health Organization, 2004; Kanis et al., 2008). This criterion has now been widely accepted and provides both a diagnostic and intervention threshold. Therefore, the WHO criterion was adopted in this study.

Measure of prolactin levels. A total of $8 \mathrm{ml}$ of fasting blood sample was collected at 7:00 AM on the next day of admission from each participant and again 12 months later. Three milliliters of blood sample was used to measure serum PRL level and E2 by electrochemical luminescence method. The sample count coefficient of variation was $\leq 3.49 \%$, according to the WHO standards. In this study, we used the 601 Electrochemical Luminescence Counting Instrument manufactured by the Diagnostics Division of German Company Roche. Five milliliters of blood was used to detect serum levels of BALP and C-terminal telopeptides of type I collagen.

Study design and procedure. This is a prospective study design. All participants were invited to the clinical laboratory in Shandong Mental Health Center at both baseline and 12 months later. After blood samples were taken, their BMD was measured by X-ray absorptiometry. They were then instructed to complete a questionnaire booklet before they left the laboratory. The same measures were conducted 12 months later.

Statistics analysis. Data analysis was conducted using SAS version 9.1 (SAS Institute Inc, Cary, NC, USA). Demographic and clinical features were compared between groups using independent samples $t$-tests, continuity corrected Chi-square tests (Yates' correction) as appropriate. Comparisons of BMD value, PRL level, E2, BALP, and CTX among conventional, atypical, and control groups were conducted using paired $t$-test before and after treatment. The association between the PRL level and the BMD value after treatment in both conventional and atypical groups was examined using Pearson's correlation analysis. Multivariate logistic regression (backward stepwise) modeling was conducted to explore the effect of baseline variables on BMD measure (z-core) at 12 months.

\section{RESULTS}

\section{Demographic and clinical characteristics}

During the study, 28 patients had to switch to other antipsychotics because of poor therapeutic benefits or side effects, 33 patients dropped out because of withdrawal and loss of contact, and 16 patients needed to combine treatment with another medication. In total, 77 patients dropped out of the study, and therefore the drop-out rate was about $32.08 \%$. Of those, 39 cases dropped out from the conventional group (chlorpromazine 14 cases, perphenazine 13 cases, and sulpiride 12 cases), with a drop-out rate of $32.50 \%$; and 38 cases dropped out from the atypical group (clozapine 14 cases, quetiapine 12 cases, and aripiprazole 12 cases), with a drop-out rate of $31.66 \%$. The drop-out rates were roughly equivalent for conventional and atypical antipsychotics, and consistent with that reported in a meta-analysis, which indicates that drop-out rates exceed a third of patients treated with antipsychotic medication in clinical trials (Wahlbeck et al., 2001; Lieberman et al., 2005). A total of 163 patients completed the study, including 81 cases in the conventional group and 82 cases in the atypical group. Demographic and clinical characteristics are presented in Table 1. There were no significant differences between the patient group (including both the conventional group and the atypical group) and the healthy control group in terms of age, BMI and education, gender, and marital status ( $p>0.05$ in all cases). In addition, there were no significant differences between patients in the conventional group and the atypical group in terms of age, BMI, total score of the PANSS, education, disease duration, gender, and subtypes of schizophrenia $(p>0.05$ in all cases, see Table 1).

\section{Comparison of bone mineral density measure between groups}

There were no significant differences of BMD values of PA L1-L4 between the control group and the two patient groups (conventional and atypical group) before 
Table 1. Comparison of demographic and clinical characteristics

\begin{tabular}{|c|c|c|c|}
\hline & Conventional $(n=81)$ & Atypical $(n=82)$ & Control $(n=90)$ \\
\hline Age (years, mean $\pm \mathrm{SD})$ & $35.9 \pm 11.1$ & $33.1 \pm 10.3$ & $34.2 \pm 10.6$ \\
\hline Sex (male) & $43(53 \%)$ & $43(52 \%)$ & $47(52 \%)$ \\
\hline Age of onset (years, mean \pm SD) & $27.0 \pm 8.9$ & $26.2 \pm 9.7$ & \\
\hline Duration of illness (years, mean $\pm S D$ ) & $0.7 \pm 0.4$ & $0.6 \pm 0.5$ & \\
\hline Education (years, mean \pm SD) & $12.9 \pm 3.11$ & $12.1 \pm 2.3$ & \\
\hline \multicolumn{4}{|l|}{ Subtype of schizophrenia } \\
\hline Other schizophrenia & $7 \%$ & $7 \%$ & \\
\hline \multicolumn{4}{|l|}{ PANSS total score $($ mean \pm SD) } \\
\hline Before treatment & $86.7 \pm 17.5$ & $87.4 \pm 18$ & \\
\hline After treatment & $50.4 \pm 13$ & $44.4 \pm 13.7$ & \\
\hline
\end{tabular}

$n$, number of participants. Student's $t$-tests were performed between the following: the conventional group versus the atypical group, the conventional group versus the control group, and the atypical group versus the control group ( $p>0.05$ in all cases).

PANSS, Positive and Negative Symptoms Scale.

treatment $(p>0.05)$. However, the BMD value of the conventional group was significantly lower than that of the control group at 12 months after treatment, whereas there was no difference between the atypical group and the control group $(p>0.05)$ (see details in Table 2).

In comparison with pre-treatment, the BMD value in the conventional group decreased significantly after treatment; the BMD value in the atypical group decreased slightly at 12 months after treatment but failed to reach any significance $(p>0.05)$. When comparing the BMD value of the conventional group to the atypical group, there was no significant difference before treatment $(p>0.05)$, whereas the BMD value of the conventional group was significantly lower than that of the atypical group at 12 months after treatment.

The study also compared incidence rates of osteoporosis in the patient group and the control group at pre-treatment baseline and 12 months after treatment and at $\mathrm{L} 1-\mathrm{L} 4$, respectively. The finding indicates that the patient group had a significantly higher osteoporosis incidence rate than the control group at 12 months after treatment across PA L1-L4 level $\left(\chi^{2}=18.793 \sim 31.199, p<0.05\right.$ in all cases $)$.

Comparison of prolactin levels, estrogen, bone alkaline phosphatase, and crosslaps between groups

The study also compared the values of PRL, E2, BALP, and CTX between conventional and atypical groups, before and after treatment. In the conventional group, the PRL after treatment was significantly higher, and both E2 and BALP were significantly lower than that before treatment; however, there were no significant treatment effects on PRL, E2, BALP, and CTX in the atypical group, details as shown in Table 3.

Table 2. Comparison of bone mineral density measure $\left(\mathrm{g} / \mathrm{cm}^{2}\right)$ between patients and controls

\begin{tabular}{lcccrrrr}
\hline Lumbar & Conventional $(n=81)$ & Atypical $(n=82)$ & Controls $(n=90)$ & $t_{1}\left(p_{1}\right)$ & $t_{2}\left(p_{2}\right)$ & $t_{3}\left(p_{3}\right)$ & $t_{4}\left(p_{4}\right)$ \\
\hline L1 before & $1.08 \pm 0.16$ & $1.04 \pm 0.17$ & $1.16 \pm 0.12$ & -3.714 & -5.437 & $-3.938^{*}$ & -0.549 \\
after & $1.05 \pm 0.07$ & $1.02 \pm 0.15$ & $1.15 \pm 0.12$ & $(0.09)$ & $(0.12)$ & $(0.009)$ & $(0.512)$ \\
L2 before & $1.19 \pm 0.18$ & $1.12 \pm 0.17$ & $1.24 \pm 0.12$ & -3.172 & -5.389 & $-3.419^{*}$ & -0.553 \\
after & $1.13 \pm 0.11$ & $1.10 \pm 0.19$ & $1.25 \pm 0.12$ & $(0.06)$ & $(0.14)$ & $(0.014)$ & $(0.647)$ \\
L3 before & $1.25 \pm 0.17$ & $1.14 \pm 0.17$ & $1.42 \pm 1.23$ & -1.672 & -0.714 & $-1.476^{*}$ & -1.947 \\
after & $1.20 \pm 0.12$ & $1.15 \pm 0.16$ & $1.42 \pm 1.36$ & $(0.17)$ & $(0.35)$ & $(0.048)$ & $(0.502)$ \\
L4 before & $1.26 \pm 0.19$ & $1.14 \pm 0.15$ & $1.28 \pm 0.15$ & -0.916 & -1.982 & $-2.452^{*}$ & -0.832 \\
after & $1.23 \pm 0.10$ & $1.16 \pm 0.18$ & $1.28 \pm 0.13$ & $(0.39)$ & $(0.08)$ & $(0.03)$ & $(0.767)$ \\
\hline
\end{tabular}

$t_{1}\left(p_{1}\right)$, the conventional group before treatment versus the control group at baseline.

$t_{2}\left(p_{2}\right)$, the atypical group before treatment versus the control group at baseline.

$t_{3}\left(p_{3}\right)$, the conventional group versus the control group 12 months after treatment.

$t_{4}\left(p_{4}\right)$, the atypical group versus the control group 12 months after treatment.

L1, lumbar vertebra1; L2, lumbar vertebra 2; L3, lumbar vertebra 3; L4, lumbar vertebra 4.

${ }^{*} p<0.05$. 
Correlation between prolactin levels, estrogen, bone alkaline phosphatase, crosslaps, and bone mineral density in treatment groups

Results from the correlation analysis among BMD and PRL, E2, BALP, CTX in both conventional and atypical groups are shown in Table 4 . In the conventional group, the BMD value was negatively correlated with PRL and CTX across L1-L4 at 12 months after treatment, whereas a positive correlation was found between E2, BALP, and BMD $(p<0.05)$. However,

Table 3. Comparison of prolactin levels, estrogen, bone alkaline phosphatase, and crosslaps between treatment groups

\begin{tabular}{llcl}
\hline & & Conventional $(n=81)$ & Atypical $(n=82)$ \\
\hline PRL & Before & $31.73 \pm 22.18$ & $29.79 \pm 16.03$ \\
$(\mathrm{ng} / \mathrm{ml})$ & After & $53.05 \pm 30.25^{\mathrm{a} *}$ & $32.81 \pm 17.42^{\mathrm{b}} *$ \\
E2 & Before & $45.23 \pm 10.77$ & $47.02 \pm 14.31$ \\
(U/l) & After & $22.87 \pm 12.14^{\mathrm{a} *}$ & $45.99 \pm 17.19^{\mathrm{b}} *$ \\
BALP & Before & $26.32 \pm 5.48$ & $27.78 \pm 4.97$ \\
$(\mathrm{ng} / \mathrm{ml})$ & After & $20.17 \pm 5.34^{\mathrm{a} *}$ & $28.10 \pm 5.19^{\mathrm{b}} *$ \\
CTX & Before & $0.45 \pm 0.23$ & $0.50 \pm 0.19$ \\
(U/l) & After & $0.50 \pm 0.12$ & $0.55 \pm 0.12$ \\
\hline
\end{tabular}

PRL, prolactin level; E2, estrogen; BALP, bone alkaline phosphatase; CTX, crosslaps.

${ }^{a}$ The conventional group before treatment versus after treatment.

${ }^{\mathrm{b}}$ The conventional group versus the atypical group after treatment. $* p<0.05$. there was no significant correlation between PRL, $\mathrm{E} 2, \mathrm{BALP}, \mathrm{CTX}$, and BMD in the atypical group $(p>0.05)$.

\section{Multivariate logistic regression analysis}

Age, gender, BMI, duration of illness, education, PANSS score, antipsychotic treatments (conventional or atypical), PRL, E2, BALP, and CTX were entered as covariates into a multivariate logistic regression model using backward stepwise method, with BMD $z$-score (derived from the ratio of patients BMD measure to age-matched healthy controls' BMD measure) at 12 months as the dependent variable (Table 5). The use of conventional antipsychotics, PRL, and CTX was entered into the regression equation and remained significant in the resultant model.

\section{DISCUSSION}

This study investigated how long-term use of antipsychotics affects BMD and how this is associated with PRL in patients with schizophrenia. The study compared effects of two generations of antipsychotics (both conventional and atypical) on BMD and its association with PRL at baseline and 12 months after treatment. The study found that the BMD value of patients taking conventional antipsychotics (chlorpromazine, perphenazine, and sulpiride)

Table 4. Correlations between bone mineral density and prolactin levels, estrogen, bone alkaline phosphatase, crosslaps from the conventional and atypical groups before and after treatment

\begin{tabular}{|c|c|c|c|c|c|c|c|c|c|}
\hline & & \multicolumn{4}{|c|}{ Conventional group $(n=81)$} & \multicolumn{4}{|c|}{ Atypical group $(n=82)$} \\
\hline & & PRL & E2 & BALP & CTX & PRL & E2 & BALP & CTX \\
\hline & After & $-0.981^{*}$ & $-0.784 *$ & $-0.323 *$ & $-0.731^{*}$ & 0.410 & -0.305 & -0.493 & 0.472 \\
\hline L2 & Before & -0.061 & 0.024 & 0.013 & 0.037 & -0.074 & -0.075 & -0.031 & 0.316 \\
\hline & After & $-0.812^{*}$ & $-0.543 *$ & $-0.069 *$ & $-0.931 *$ & 0.419 & -0.269 & 0.532 & 0.219 \\
\hline L3 & Before & 0.028 & 0.435 & 0.023 & 0.328 & -0.026 & -0.112 & -0.301 & -0.421 \\
\hline & After & $-0.567 *$ & $0.505^{*}$ & $-0.997 *$ & $0.837 *$ & 0.315 & -0.409 & 0.480 & 0.473 \\
\hline
\end{tabular}

PRL, prolactin level; E2, estrogen; BALP, bone alkaline phosphatase; CTX, crosslaps. $* p<0.05$.

Table 5. Logistic regression analysis of predictors for post-treatment bone mineral density $z$-score

\begin{tabular}{lrrrr}
\hline Model & B & SE & Beta & \\
\hline Constant & -3.410 & 4.571 & -0.703 \\
Conventional antipsychotics & 0.621 & 0.149 & -0.422 & -4.917 \\
PRL & -1.642 & 0.541 & -0.339 & $-0.000^{* *}$ \\
CTX & -0.001 & 0.021 & $0.004 *$ \\
\hline
\end{tabular}

PRL, prolactin level; CTX, crosslaps.

$* p<0.05$.

$* * p<0.001$ 
decreased significantly 12 months after treatment, and that this is significantly correlated with PRL level, whereas no significant change was found in patients taking atypical antipsychotics (quetiapine, risperdal, and clozapine). The study also indicates that the incidence rate of osteoporosis $(11.4 \sim 21.7 \%)$ across L1-L4 increased significantly 12 months after conventional antipsychotic treatment in patients with schizophrenia, and this was significantly higher than that of healthy controls $(0 \sim 1.6 \%)$.

One of the currently accepted hypotheses for the pathogenesis of schizophrenia is the hyperactivity of dopamine and serotonin (5-HT) function. PRL secretion is regulated by both the prolactin release factor and the prolactin inhibitory factor (PIF). Serotonin (5-HT) receptors can promote prolactin release factor secretion, whereas dopamine receptors promote PIF secretion. Normal level of PRL is under this dual control. Antipsychotic drugs tend to block dopamine D2 receptors, which attenuates the inhibitory effect of dopamine on prolactin release, leading to hyperlactinemia. Typical antipsychotics are not particularly selective and also block dopamine receptors in the mesocortical, tubero-infundibular, and the nigrostriatal pathways. Atypical antipsychotic drugs have a similar blocking effect on D2 receptors, and some also block or partially block serotonin receptors (particularly $5-\mathrm{HT}_{2 \mathrm{~A}}, \mathrm{C}$ and $5-\mathrm{HT}_{1 \mathrm{~A}}$ receptors). However, atypical antipsychotics appear not to block dopamine within the tubero-infundibular tract, a dopamine pathway that also controls prolactin secretion (Emiliano and Fudge, 2004; Hummer and Huber, 2004). The current study indicated a significantly higher level of PRL after long-term use of conventional antipsychotics but not atypical antipsychotics. The lack of prolactin elevation reported with the atypical antipsychotics may be due to their much greater specificity, which results in fewer blockades of dopamine receptors in the tubero-infundibular pathway and some of their effects on 5- $\mathrm{HT}_{2}$ receptors (Petty, 1999; Halbreich and Kahn, 2003; Kishimoto et al., 2005; Peveler et al., 2008; Walters and Jones, 2008; Riecher-Rössler et al., 2009). In addition, perhaps a dynamic balance between bone formation and bone resorption is essential. Basic and clinical studies have shown that E2 can prevent bone loss and reduce the incidence of bone fractures, and the underlying basis of this protective effect involves the prevention of apoptosis in osteoblasts and the inhibition of caspase-3 activity (Ataya et al., 1988; Bradford et al., 2010).

Kameda et al. (1997) found that E2 inhibited hydrogen ion release, which may directly suppress the absorption functions of osteoclasts. Goodman et al. (2007) also reported that the incidence of osteoporosis may be related to E2 level. It has been proposed that the beneficial effects of E2 are due to the ability of E2 to suppress osteoclastogenic cytokine production in T-cells and osteoblasts and to induce the apoptotic death of osteoclasts (Krum and Brown, 2008). However, because of lack of large sample size and lack of longitudinal design, the association between E2 and osteoporosis is not yet fully unraveled.

Bone alkaline phosphatase is a biochemical index of bone formation, whereas CTX is a biochemical index of bone absorption. In this study, serum BALP level in the conventional antipsychotic group reduced significantly after treatment, whereas CTX level increased significantly after treatment. There were no changes of either BALP or CTX level in the atypical antipsychotic group.

Findings from this study suggest that long-term use of conventional antipsychotics produces treatment effects by blocking dopamine D2 receptors; however, they also interfere with the endocrine system and metabolism. The inhibition of the hypothalamus on the pituitary gland may decrease PIF and E2 secretion and increase PRL secretion. This then affects the activity of bone cells, causing increased osteoclast activity, reduced bone formation, increased bone absorption, and unbalanced reconstruction, resulting in reduced bone mineral density and causing osteoporosis.

Although the idea that antipsychotics may lead to osteoporosis through the development of hyperprolactinemia has been studied before, the strength of current study is that it investigates patients with first-episode psychosis using a 12-month prospective cohort design. The further strength is the random allocation of treatments. Given that very few studies that longitudinally examined the impact of antipsychoticinduced hyperprolactinemia on bone metabolism, this study is the largest compared with earlier evidence; therefore, the findings from this study are very important. One limitation of the study is the lack of clinical assessment of more risk factors for osteoporosis rather than age, gender, and BMI, which should be assessed in future research. The findings of the current study are consistent with those of Meaney et al. (2004) who found that patients with schizophrenia on long-term antipsychotic medication are at high risk of developing reduced bone mineral density and suggested that this may be a consequence of hyperprolactinemia-induced hypogonadism. The study provides further research evidence on this and suggests that the increase of PRL level is an important risk factor leading to osteoporosis after long-term use of some conventional antipsychotics. In comparison, most atypical antipsychotics do not affect PRL level. Therefore, when prescribing antipsychotics for long-term use in chronic schizophrenia, the awareness of an elevated risk of drug-induced osteoporosis is beneficial for both 
clinicians and patients. However, decreased BMD and osteoporosis are multifactorial processes, and abnormal bone structure and functions are not limited to BMD (Halbreich, 2007). Multiple dynamic processes may lead to the impairment of bone homeostasis. Future research using comprehensive and multifactorial approaches is warranted to develop relevant treatment and prevention strategies.

\section{CONFLICTS OF INTEREST}

The authors have declared no conflict of interest.

\section{ACKNOWLEDGEMENT}

The project is funded by the Medicine and Health Technology Development program of Shandong Province (grant reference number: 2007HZ08).

\section{REFERENCES}

Alldredge BK, Kimble K, Anne M, Lloyd Y, Kradjan WA, Guglielmo BJ. 2009. Applied Therapeutics: The Clinical use of Drugs. Wolters Kluwer Health/Lippincott Williams \& Wilkins: Philadelphia.

Ataya K, Mercado A, Kartaginer J, Abbasi A, Moghissi KS. 1988. Bone density and reproductive hormones in patients with neuroleptic-induced hyperprolactinemia. Fertil Steril 50: 876-881.

Bradford PG, Gerace KV, Roland RL, Chrzan BG. 2010. Estrogen regulation of apoptosis in osteoblasts. Physiol Behav 99: 181-185.

Emiliano AB, Fudge JL. 2004. From galactorrhea to osteopenia: rethinking serotonin-prolactin interactions. Neuropsychopharmacology 29: 833-846.

Goodman SB, Jiranek W, Petrow E, Yasko AW. 2007. The effects of medications on bone. J Am Acad Orthop Surg 15: 450-460.

Graham SM, Howgate D, Anderson W, et al. 2011. Risk of osteoporosis and fracture incidence in patients on antipsychotic medication. Expert Opin Drug Saf 10: 575-602.

Halbreich U. 2007. Osteoporosis, schizophrenia and antipsychotics: the need for a comprehensive multifactorial evaluation. CNS Drugs 21: 641-657.

Halbreich U, Kahn LS. 2003. Hyperprolactinemia and schizophrenia: mechanisms and clinical aspects. J Psychiatr Pract 9: 344-353.

Howard L, Kirkwood G, Leese M. 2007. Risk of hip fracture in patients with a history of schizophrenia. Br J Psychiatry 190: 129-134.

Hummer M, Huber J. 2004. Hyperprolactinaemia and antipsychotic therapy in schizophrenia. Curr Med Res Opin 20: 189-197.

Javaid MK, Holt RI. 2008. Understanding osteoporosis. J Psychopharmacol 22: $38-45$.

Kameda T, Mano H, Yuasa T, et al. 1997. Estrogen inhibits bone resorption by directly inducing apoptosis of the bone-resorbing osteoclasts. $J$ Exp Med 186: 489-495.

Kaneda Y, Kawamura I, Fujii A, Ohmori T. 2004. Impact of a switch from typical to atypical antipsychotic drugs on quality of life and gonadal hormones in male patients with schizophrenia. Neuro Endocrinol Lett 25: $135-140$.

Kanis JA, McCloskey EV, Johansson H, Oden A, Melton LJ 3rd, Khaltaev N. 2008. A reference standard for the description of osteoporosis. Bone 42: 467-475.

Kay SR, Opler LA, Lindenmayer JP. 1988. Reliability and validity of the positive and negative syndrome scale for schizophrenics. Psychiatry Res 23: 99-110.
Kishimoto T, Watanabe K, Takeuchi H, et al. 2005. Bone mineral density measurement in female inpatients with schizophrenia. Schizophr Res 77: $113-115$

Krum SA, Brown M. 2008. Unraveling estrogen action in osteoporosis. Cell Cycle 7: 1348-1352.

Lee TY, Chung MY, Chung HK, Choi JH, Kim TY, So HS. 2010. Bone density in chronic schizophrenia with long-term antipsychotic treatment: preliminary study. Psychiatry Investig 7: 278-284.

Lieberman JA, Stroup TS, McEvoy JP. 2005. Effectiveness of antipsychotic drugs in patients with chronic schizophrenia. N Engl J Med 353: 1209-1223.

McGrath J, Saha S, Chant D, Welham J. 2008. Schizophrenia: a concise overview of incidence, prevalence, and mortality. Epidemiol Rev 30: 67-76.

Meaney AM, Smith S, Howes OD, O'Brien M, Murray RM, O'Keane V. 2004. Effects of long-term prolactin-raising antipsychotic medication on bone mineral density in patients with schizophrenia. Br J Psychiatry 184: 503-508.

Molitch ME. 2005. Medication-induced hyperprolactinemia. Mayo Clin Proc 80: 1050-1057.

Montejo AL. 2008. Prolactin awareness: an essential consideration for physical health in schizophrenia. Eur Neuropsychopharmacol 18: S108-S114.

Montgomery J, Winterbottom E, Jessani M, et al. 2004. Prevalence of hyperprolactinemia in schizophrenia: association with typical and atypical antipsychotic treatment. J Clin Psychiatry 65: 1491-1498.

Partti K, Heliövaara M, Impivaara O, et al. 2010. Skeletal status in psychotic disorders: a population-based study. Psychosom Med 72: 933-940.

Petty RG. 1999. Prolactin and antipsychotic medications: mechanism of action. Schizophr Res 35: S67-S73.

Peveler RC, Branford D, Citrome L, et al. 2008. Antipsychotics and hyperprolactinaemia: clinical recommendations. J Psychopharmacol 22: 98-103.

Renn JH, Yang NP, Chueh CM, Lin CY, Lan TH, Chou P. 2009. Bone mass in schizophrenia and normal populations across different decades of life. BMC Musculoskelet Disord 10: 1.

Renn JH, Yang NP, Chou P. 2010. Effects of plasma magnesium and prolactin on quantitative ultrasound measurements of heel bone among schizophrenic patients. BMC Musculoskelet Disord 11: 35.

Rey-Sánchez P, Lavado-García JM, Canal-Macías ML, Gómez-Zubeldia MA, Roncero-Martín R, Pedrera- Zamorano JD. 2009. Ultrasound bone mass in schizophrenic patients on antipsychotic therapy. Hum Psychopharmacol 24: 49-54.

Riecher-Rössler A, Schmid C, Bleuer S, Birkhäuser M. 2009. Antipsychotics and hyperpolactinaemia: pathophysiology, clinical relevance, diagnosis and therapy. Neuropsychiatr 23: 71-83.

Roke Y, van Harten PN, Boot AM, Buitelaar JK. 2009. Antipsychotic medication in children and adolescents: a descriptive review of the effects on prolactin level and associated side effects. J Child Adolesc Psychopharmacol 19: 403-414.

Sugawara N, Yasui-Furukori N, Fujii A, et al. 2011. No association between bone mass and prolactin levels among patients with schizophrenia. Hum Psychopharmacol 26: 596-601.

Van Os J, Kapur S. 2009. Schizophrenia. Lancet 374: 635-645.

Wahlbeck K, Tuunainen A, Ahokas A, Leucht S. 2001. Dropout rates in randomized antipsychotic drug trials. Psychopharmacology (Berl) 155: 230-233.

Walters J, Jones I. 2008. Clinical questions and uncertainty-prolactin measurement in patients with schizophrenia and bipolar disorder. $J$ Psychopharmacol 22: 82-89.

World Health Organization. 1994. Assessment of fracture risk and its application to screening for postmenopausal osteoporosis. Report of WHO Study Group.

World Health Organization. 2004. World Health Organization scientific group on the assessment of osteoporosis at the primary health care level. Summary Meeting Report. Brussels.

World Health Organization. 2011. Schizophrenia. Available at: (http://www.who. int/mental_health/management/schizophrenia/en/) Assessed 26 February 2013 